\title{
La paradoja patagónica frente a las políticas nacionales y globales ${ }^{1}$
}

\author{
Antonio Daher ${ }^{2}$
}

\begin{abstract}
RESUMEN
La Región de Aysén es un caso paradigmático y, a la vez, paradójico. Siendo una "región extrema" es, sin embargo, la única al sur de Coquimbo que aumenta su participación en el PIB nacional en los últimos cuarenta años. Es más, Aysén crece un 28\% en contraste a Magallanes, que es la que más reduce -en un $54.5 \%$ - su participación en el PIB del país: ¡una paradoja entre dos regiones patagónicas! Esta es la cuestión a investigar. En las conclusiones, se constata un sostenido sesgo pro norte y antisur del modelo de economía abierta basado en el sector exportador de recursos naturales. Más aún, se verifica que, aunque en la media nacional crecen fuertemente los sectores terciarios-urbanos, las "regiones ganadoras" poseen una acentuada "reprimarización" que fundamenta que se las pueda identificar como "regiones-commoditity", más plenamente insertas en los mercados globales. Detrás de este modelo están las políticas gubernamentales de libre mercado, apertura económica, desgravación arancelaria, promoción exportadora e inserción global, además de las políticas monetarias y cambiarias. Y también las de los organismos supranacionales de la gobernanza económica global, que no solo afectan a los países y sus gobiernos, sino muy diferencialmente a sus regiones subnacionales.
\end{abstract}

Palabras clave: Geopolítica financiera, políticas macroeconómicas, regiones-commodity, Patagonia.

\begin{abstract}
The Aysen Region is both a paradigmatic and paradoxical case. As an "extreme region", it is the only region in Chile south of Coquimbo that has increased its contribution to national GDP in the past forty years. Additionally, Aysen grew by $28 \%$ compared to Magallanes, the region that experienced the most significant decrease in contribution to the national GDP (54.5\%); a paradox between two Patagonian regions! This is the research question. Finding show that there is a clear pro-north and anti-south bias within the open economy model, based on the natural resource exportation sector. Furthermore, it can be confirmed that at a national level, while the tertiary-urban sectors have experienced significant growth, the "winning regions" have experienced an accentuated "re-primarization". This establishes the basis for what can be identified as "commodity-regions", which are more fully embedded within global markets. Aysen, with a relatively late-blooming salmon industry and a renewed mining sector, would seem to be taking on and joining this primary-export model. Behind this model are a number of factors such as free market policies, economic liberalization, tariff exemptions and deductions, export promotion and global insertion, in addition to corresponding monetary and exchange policies. Supranational organizations involved in global economic governance must also be taken into account, as the macroeconomic policies implemented at this scale affect not only countries and their national governments, but also have a differential impact on sub-national regions as well.
\end{abstract}

Key words: Financial geopolitics; macroeconomic policies; commodity-regions; Patagonia

1 CEDEUS, CONICYT/ FONDAP 15110020 y FONDECYT 1150636. Artículo recibido el 23 de diciembre de 2014, aceptado el 22 de junio de 2015 y corregido el 29 de julio de 2015.
2 Profesor Titular, Pontificia Universidad Católica de Chile (Chile).E-mail: adaher@uc.cl 
La paradoja patagónica se perfila claramente al confrontar, en los últimos cuarenta años - periodo correspondiente en términos generales al del nuevo modelo de desarrollo de economía de mercado o 'neoliberal'- las similitudes y diferencias sustantivas en los procesos económicos y cambios en la estructura del producto interno bruto de las dos regiones más australes de Chile, Aysén y Magallanes. Tales cambios estructurales revelan tanto el fuerte impacto de las políticas públicas explícitamente no territoriales o formuladas a-espacialmente (macroeconómicas, de apertura, fomento exportador e inserción global) como la acusada presencia estatal -según se verificará más adelanteen ambas regiones de frontera.

Serán sin embargo estas políticas no territoriales -más que las regionales, de descentralización o las específicas para las regiones extremas- las que explicarán los comportamientos distintos y prácticamente opuestos de las dos regiones, una nítidamente "ganadora", junto a las mineras del Norte Grande y Chico, y la otra la más rezagada y "perdedora" a nivel nacional.

Por lo demás, estas regiones patagónicas comparten con algunas del norte la condición de territorios extremos y fronterizos y por ende, participan similarmente de algunos de los beneficios y subsidios de las políticas estatales formuladas para favorecer dichos territorios: los resultados -explicables no por ellas sino por las políticas macro aespaciales- no pueden ser más diferentes: un norte donde la norma es el éxito económico, aunque con variaciones inter e intrarregionales, y un sur austral donde solo la excepción -Aysén- tiene ese éxito en medio de un panorama generalizado de decrecimiento relativo. Todo lo anterior, ciertamente en las regiones perdedoras pero también en las regiones ganadoras, coexistiendo con innegables desigualdades sociales y territoriales a la vez que con una significativa deuda en términos de sustentabilidad y calidad de vida.

En cuanto al objetivo de investigación, este artículo se propone explicar el muy disímil crecimiento de dos regiones contiguas y extremas y relevar el rol estatal y supraestatal de las políticas nacionales y supranacionales no territoriales -macroeconómicas y financieras- en la predeterminación de la configuración geoeconómica de las regiones, con especial foco en los casos de Aysén y Magallanes.

Con el propósito de cumplir el objetivo descrito, y junto a una amplia revisión de la literatura especializada y la elaboración de un marco conceptual ad hoc, se contempla el uso de información secundaria y el análisis estadístico de datos económicos, específicamente del PIB regional y sectorial-regional (Banco Central de Chile, 2012 y 2013; Observatorio Regional SUBDERE, 2013; Guerrero y Pozo, 2012) para el periodo 1970-2010, es decir para cuatro décadas que, además de ser representativas de tendencias, corresponden al cambio de modelo económico en el país. La investigación se focaliza en el estudio comparado de dos casos, correspondientes a dos regiones patagónicas contiguas que, aunque con similitudes geográficas, presentan dinámicas económicas muy opuestas.

\section{La paradoja patagónica}

¿Cómo comprender esta doble paradoja de la Región de Aysén, es decir, que resulte ser la única entre todas las regiones del centro, sur y australes del país que aumente su participación en el PIB nacional en cuatro décadas $y$, a la vez, que presente un dinamismo económico tan distinto y de hecho diametralmente opuesto a Magallanes, su región vecina, que posee el mayor decrecimiento económico en igual periodo?

En respuesta a esta cuestión se plantea hipotéticamente que ambas regiones, inmersas en el mismo modelo económico nacional liberal -aun presentando una fuerte hipertrofia "estatista" en su sector público- siguen históricamente caminos divergentes: mientras Aysén, consecuente con el modelo primarioexportador del país, progresivamente deviene más y más en una "región-commodity", Magallanes, por el contrario -decaídas sus exportaciones originadas en hidrocarburos fósiles- va cerrando paulatinamente su economía que se hace cada vez más "doméstica".

$\mathrm{Al}$ analizar estos procesos divergentes y la consiguiente paradoja patagónica se descubren numerosas similitudes -no necesariamente equivalentes- en los procesos econó- 
micos de Aysén y Magallanes en las últimas cuatro décadas... y, sorprendentemente, solo dos muy significativas diferencias. Estas últimas darán cuenta de los derroteros opuestos de las dos regiones.

Entre las similitudes, cabe destacar en primer lugar la caída en la participación dentro del producto del sector silvoagropecuario, mucho más pronunciada en Aysén (del 25,1\% en 1970 a solo el 1,8\% en 2010) que en Magallanes (del $6,2 \%$ al $1,7 \%$ en iguales fechas). En el país, esta tendencia decreciente está representada por indicadores de $8,2 \%$ y $2,7 \%$ en los años inicial y final.

Contrariamente a la caída nacional de la industria manufacturera, de casi un cuarto del PIB en $1970(24,7 \%)$ a un 10,6\% cuatro décadas después - proceso conocido como "desindustrialización" y opuesto al anterior modelo cepalino de industrialización sustitutiva- el producto industrial incrementa su participación en Aysén, en igual periodo, de 1,2 a 4,4\%, casi cuadruplicándose, y en Magallanes de 3,6 a $15,3 \%$, casi triplicándose.

Análogamente, mientras a nivel nacional la construcción decayó levemente del 7,5 al $6,7 \%$ del producto, en Aysén creció del 14,9 al 21,2\%, y en Magallanes del 3,3 al 7,1\%, posiblemente asociado a la inversión en obras públicas, sobre todo en Aysén.

Curiosamente el turismo, crecientemente relevante en ambas regiones aunque más en Magallanes, no se refleja en los indicadores del sector comercio, restaurantes y hoteles, los que decrecen significativamente en Aysén, del 18,7 en 1970 a solo el 7,5\% en 2010, y más levemente en Magallanes, de 12,0 a 10,2\% respectivamente (la participación nacional del sector decae de 16,5 a 10,2\% en los mismos años).

El sector pesca -el cual, según se verá más adelante, resultará ser uno de los decisivos- y que a nivel país se mantiene constante en cuatro décadas, con una participación muy marginal de $0,3 \%$, crece en ambas regiones, muy fuertemente en Aysén -de 0,2 a un notable $9,1 \%$ y en menor medida en $\mathrm{Ma}$ gallanes, de 0,4 a 1,3\% en igual periodo.

El sector de servicios personales muestra crecimiento tanto en las dos regiones patagó- nicas como en la media del país. En efecto, esta última sube de 6,2 a $10,5 \%$ en cuatro décadas, como parte de la tendencia terciarizadora. En el mismo periodo, en la Región de Aysén ella pasa del 9,1 al 13,5\% -siempre sobre el promedio nacional- mientras que en Magallanes el incremento es aún mayor, de un relativamente bajo $4,4 \%$ en 1970 a un $9,6 \%$ en 2010 , más que duplicando su participación.

Sin embargo, y siempre en el sector terciario, los cambios más relevantes se observan en la administración pública: en el contexto de la implementación de una economía de mercado y subsidiariedad estatal, y por consiguiente con reducción o contención del tamaño de la administración pública, este sector en la media nacional baja de 5 a $4,2 \%$ su contribución al producto. Muy por el contrario, en Aysén sube significativamente de 8.1 a un considerable $24 \%$, triplicándose hasta representar casi un cuarto del producto regional; $y$ en Magallanes pasa del 4,4 al 14,9\%, más que triplicándose.

En síntesis, entre las similitudes observables en los cambios de la estructura económica de ambas regiones destacan, siguiendo además la tendencia nacional, la caída en la participación del sector silvoagropecuario y en la del sector comercio, restaurantes y hoteles. Asimismo, Aysén y Magallanes, alineadamente con el país, registran un incremento en los servicios personales.

En cambio, ambas regiones, a contracorriente de la tendencia del país, suben la participación de sus sectores de industria manufacturera, construcción, y pesca -en este último Chile tiene prácticamente valores invariables-. Y por cierto, como se ha dicho, ambas regiones definitivamente se apartan de la media nacional -y en sentido opuesto- en el impresionante crecimiento de su administración pública.

Sin minimizar la incidencia de estas transformaciones, ellas, con la sola excepción del sector pesca de muy desigual dinamismo, precisamente por ser compartidas entre ambas regiones australes -e independientemente de si siguen o contradicen las tendencias nacionales en los respectivos sectores- darían cuenta de un comportamiento económico 
más bien análogo en las dos. Y sucede todo lo contrario. ¿Qué explica entonces, principalmente y a pesar de estas similitudes, que Aysén crezca en cuatro décadas de modo solo comparable a las regiones del norte del país, y que sea la única entre las diez regiones del centro, sur y área austral de Chile que incrementa su participación en el producto nacional, mientras que Magallanes sea la que menos crece y más reduce su contribución a dicho producto?

La respuesta está parcialmente en uno de los sectores económicos que en ambas regiones patagónicas crece -asociado a la expansión latitudinal cada vez más austral de la salmonicultura- pero a tasas muy desiguales: la pesca. En Aysén, la contribución del sector se multiplica ni más ni menos que 57 veces en cuarenta años, en tanto que en Magallanes solo lo hace en 3,6 veces.

La respuesta está también en uno de los sectores en que ambas regiones registran de hecho procesos no solo diferentes, sino radicalmente divergentes: el sector minería. En efecto, mientras que en Aysén dicho sector casi se cuadruplica, pasando del 1,1\% en 1970 al 3,7\% en 2010, en Magallanes baja de un mayoritario $52,4 \%$ al $20,6 \%$ en los mismos años -por la minimización de la producción sustitutiva de hidrocarburos- reduciéndose de más de la mitad a solo un quinto. Mientras la Región de Aysén se suma progresivamente al éxito de la minoría de las regiones más mineras del país, Magallanes, a contracorriente del modelo primario-minero exportador, se resta del mismo, el que a nivel país pasa de un $6,6 \%$ a un $13,4 \%$, duplicando su contribución al PIB en cuarenta años.

Así pues, en tanto Aysén se va configurando progresivamente como una "región-Commodity" (Daher, 2003) con dos sectores -minería y pesca- orientados a los mercados globales, Magallanes, en cambio, deviene cada vez más en una economía más doméstica, como lo fue también la de la región menos exportadora de Chile, la Araucanía, que ha comenzado a revertir esa situación a partir de la maduración de sus inversiones forestales. La expansión de la salmonicultura magallánica, aún incipiente, podría dar inicio a una reversión de ese ciclo.

Inequívocamente ambas regiones patagónicas, contradiciendo el modelo y la tendencia nacional, se han "estatizado", con una notable hipertrofia de su administración pública, mucho mayor ciertamente en Aysén. Pero esta región, simultáneamente, se ha 'commoditizado', convirtiéndose en un 'territorio de exportación' crecientemente más inserto en los mercados globales. Estado y mercado, tradicionalmente divergentes, convergen excepcional y singularmente en Aysén.

\section{La producción estatal del territorio}

¿Cómo el Estado produce, con un mismo modelo de desarrollo, procesos y realidades económico-territoriales y socioterritoriales tan disímiles?, ¿en qué medida la producción estatal del territorio es directa o indirecta, tácita o explícita, coherente o contradictoria?, ¿qué primacía y cuáles efectos tienen las políticas macroeconómicas por sobre las sectoriales nacionales, tales como las de desarrollo regional y urbano?, ¿cuán autónomas, dependientes o reactivas suelen ser dichas políticas macro frente a los escenarios internacionales y a las directrices y políticas de los organismos supranacionales? Y, en el otro extremo ¿qué injerencia pueden tener los actores locales subnacionales ante las decisiones de las instancias de poder nacionales y globales?

Por cierto suele reconocerse la producción estatal (Brenner, 2004) -directa e indirecta- del territorio, mediante la inversión en obras públicas -como la Carretera Austral en la Patagonia- y también en educación, salud y vivienda social -aunque a veces con probados efectos territoriales centralizadores y regresivos-. Asimismo se la reconoce en la cooperación público-privada, tal como inicialmente en la salmonicultura austral, y además vía concesiones y subsidios -algunos en los territorios de frontera como los patagónicos-. Y por cierto también en la acción del Estado en planes de desarrollo regional, de zonas extremas -Aysén y Magallanes- y de desarrollo urbano.

En contraste, suele omitirse o minimizarse al Estado terrateniente -muy dominante a través de Bienes Nacionales en las regiones extremas del norte y australes de Chile-, al Estado empresario, incluso inmobiliario 
(Rajan \& Rancharam, 2011) y más reciente y globalmente al Estado financiarizador urbano -como en la crisis subprime- (Theurillat, 2009; Martin, 2011; Perlo, 2011; Aalbers, 2012; Harvey, 2012a y 2012b; Sassen, 2012) y al Estado radicalmente urbanizador -como en el actual plan de desarrollo de China-. Así, el Estado aparece desdibujado frecuentemente frente al protagonismo del mercado y los inversionistas y actores privados, tanto locales como internacionales.

No pocas veces se reivindica la acción territorial del Estado frente a neoliberalismos (Peck \& Tickell, 2002; Brenner \& Theodore, 2002a y 2002b) más o menos acentuados, como si la desregulación o regulación fueran las únicas opciones fundamentales. Se omite así -quizá porque es menos evidente- que la acción del Estado en la producción del territorio y sus asentamientos y ciudades es mucho más determinante cuando indirectamente se subproduce a través de políticas públicas a-espaciales o no territoriales (Jonas, 1999; Sassen, 2007) y menos aún urbanas, como lo son las políticas macroeconómicas (Daher, 1990; Escobar \& Repetto, 1993). Los efectos de estas, con impactos territoriales muy diferenciales y consecuencias sociales muy desiguales, pueden llegar a contradecir e incluso a minimizar o anular a las políticas, inversiones, subsidios y demás acciones propiamente regionales y urbanas.

El Estado, por lo demás, muchas veces es directamente responsable de agravar inequidades, como ha sucedido con algunas políticas de obras públicas y habitacionales; de producir efectos perversos que terminan beneficiando al sector empresarial privado, según se ha verificado con ciertos subsidios; o de ser definitivamente cómplice de crisis, malas prácticas y e incluso de corrupción, como lamentablemente se constata en ciertos países.

Así, la producción estatal del territorio y de la ciudad, lejos de corregir su producción mercantil, concluye confirmando que la producción social del espacio geográfico es menos dicotómica o dualista, y más sutil, compleja y contradictoria.

Más invisible y menos reconocida resulta ser la producción estatal del territorio a través de la opción por cambios en los modelos de desarrollo -como en Chile en la década de 1970- que modifican la asignación sectorial y territorial de recursos, alterando la movilidad laboral y demográfica (Sassen, 2003) según se verifica en el norte minero y en Chiloé con los acuicultivos, o desactivando los polos de desarrollo industrial en ciudades como Valparaíso y Concepción.

Menos reconocida es también la producción estatal geoeconómica (Sheppard, 2011; Sokol, 2013) y geosocial mediante políticas públicas supuestamente neutras territorialmente, como las monetarias (Leyshon, 1997; Martin, 1998; Bravo et al., 2003), cambiarias (Daher, 1990; Beine \& Coulombe, 2003) y demás políticas macroeconómicas, las que sin embargo resultan tener fuertes impactos geográficamente discriminatorios, favoreciendo por ejemplo más a las regiones exportadoras -como las mineras-y menos a las de economía doméstica- como La Araucanía y Magallanes- al punto de suscitar movimientos sociales urbanos (Sassen, 2010; Harvey, 2013) y protestas regionalistas tales como las más recientes en Aysén y las anteriores en Magallanes.

En efecto, y a pesar del relativo éxito económico de Aysén, en 2012 estalla un importante conflicto con múltiples protestas, liderado por el Movimiento Social por Aysén, que incluye, entre varias organizaciones gremiales, significativamente a los representantes del sector pesca y a la Asociación Nacional de Empleados Fiscales (ANEF) -ambos, según se ha descrito estadísticamente, de gran relevancia regional-. Entre las variadas reivindicaciones, el Movimiento reclama por la menor captura local de valor de las empresas en la zona. En Magallanes, a su vez, fuertes protestas se habían desencadenado por el alza en el precio del gas natural -fundamental en el consumo y producción de la región austral- las que Ilevaron incluso a la renuncia del ministro de Energía: el alza se debía a la Empresa Nacional del Petróleo (ENAP), una repartición del Estado. En 2013, surgía el proyecto de una nueva expresión política regionalista, el Partido Democracia Regional Patagónica, tal como ya en 1931 emergía tempranamente el Partido Regionalista de Magallanes. 
A propósito de estos movimientos sociales, Castells (2013: 209) recuerda que "a lo largo de la historia, los movimientos sociales han sido, y siguen siendo, las palancas del cambio social", valorando su dimensión local y, contemporáneamente, su amplificación global a través de las redes virtuales en la era de internet. González (2007:35), a su vez, alude a las corrientes que plantean que en los "procesos de reproducción, centralización y concentración del capital (...) es imposible explicar una colectividad local en sí misma o por sí misma, sino que en la ligazón que esa colectividad tiene con el resto del sistema internacional, particularmente en lo económico...". El mismo autor agrega: "los Estados Nacionales han sido caracterizados ya sea como víctimas o como promotores de la globalización" (González, 2007: 69), aunque aquí se sostiene más bien que los roles inherentes a estas caracterizaciones no son excluyentes en los países emergentes, alternándose en épocas de bonaza y crisis, como durante y después del denominado superciclo de los commodities.

\section{La producción supraestatal del territorio}

Pero la producción estatal del territorio - directa o indirecta, explícita o tácita, por acción u omisión- no puede ser comprendida, en medio de la globalización, sino complementada e interrelacionada con la producción supraestatal de aquel. Sin embargo, apenas se intuye la producción supranacional del territorio (Brenner, 1999; Brenner \& Elden, 2009) y las nuevas geografías (González, 2005), no solo por las corporaciones transnacionales, sino también por las políticas de los organismos internacionales tales como la Organización para la Cooperación y el Desarrollo Económico (OCDE), la Organización Mundial de Comercio (OMC), el Fondo Monetario Internacional (FMI) el Banco Mundial (BM), el Banco Central Europeo (BCE), el Banco Interamericano de Desarrollo (BID), entre otros. O simplemente por la acción de algunas instituciones que, aunque nacionales, tienen una determinante influencia global, como el Federal Reserve System (2012) -alias la FED- de los Estados Unidos.
Estos organismos resultan muy influyentes, en particular para las economías de menor tamaño plenamente insertas en los mercados globales, como la chilena (por ejemplo, el fortalecimiento del dólar ligado al alza de la tasa de interés anunciado por la FED y la consecuente devaluación de las monedas de numerosos países, entre ellas el peso chileno). Y los efectos territoriales de sus políticas se hacen más evidentes en tiempos de crisis, alterando las tasas de interés y la orientación geográfica de los flujos de capitales (Corpataux et al., 2009), como también los tipos de cambio y la competitividad de los países y sus regiones.

Sus políticas pueden resultar decisivas en el contagio geográfico de las crisis (Harvey, 2012a; Daher, 2013) o en su mitigación, como asimismo en el endoso (Harvey, 2007) o externalización internacional e interregional de muchos de sus costos económicos, sociales e incluso políticos (Daher, 2014).

Otro tanto sucede con la producción transnacional -empresarial y supraestataldel territorio, no solo local y urbano (Sassen, 2007; Manyka et al., 2012), también regional y geográficamente global. A las operaciones internacionales de inversión externa directa (en Chile, principalmente vía DL 600) y demás fondos de inversión, incluidos los soberanos, se asocia la total globalización de la banca y las finanzas (Dymsky, 2009; Alessandrini et al., 2010). A la competencia o colusión interempresarial a nivel mundial se suma la competencia o "alianza" entre naciones. A la integración regionalista, los acuerdos y tratados de libre comercio, las uniones aduaneras, las zonas monetarias (McKinnon, 1963; Kennen, 1969) -como la Eurozona- (Rodríguez-Pose \& Petrakos, 2004; Van Overtveldt, 2012; Carriére-Swalow \& Claro, 2013), y otros esfuerzos de cooperación internacional se oponen las guerras de divisas -que afectan diferencialmente a las regiones subnacionales a través del tipo de cambio, como en el "síndrome holandés" (Corden \& Neary, 1982) o el síndrome chileno del cobre (Álvarez \& Fuentes, 2006; Pereira et al., 2009)-.

También se oponen la estatización y externalización internacional de los costos de las crisis -cuyo contagio territorial es desigual según la vulnerabilidad y resilien- 
cia de las distintas economías regionales-. Especialmente se oponen la geopolítica financiera -que desplaza geográficamente (Harvey, 1982) y endosa las crisis a los países y regiones subnacionales más vulnerables-; y el riesgo moral (moral hazard) asumido por los gobiernos y organismos internacionales, cuya cuenta la "paga Moya" -según nuestro chilenismo- es decir y en último término las sociedades más vulnerables y los más pobres e indefensos: los peyorativamente denominados "países periféricos" europeos y los del tercer mundo y, al interior de ellos, las regiones y sectores más marginales y los sectores sociales más carenciados y débiles. Es la cuenta de la crisis que ahora pagan países como Brasil, Turquía, India y Chile, y muchas de sus regiones-commodity, donde emergen, aquí y allá, movimientos sociales locales detonados, sin embargo, globalmente.

\section{Conclusiones}

En consecuencia, y a modo de síntesis, corresponde replantear, en este contexto de producción estatal y supraestatal de los territorios a través de las acciones y políticas públicas nacionales e internacionales, la realidad paradójica de esas dos regiones del extremo más austral del mundo, territorios de frontera insertos sin embargo -a través de múltiples mediaciones- en la economía y gobernanza planetarias.

Así pues, y a partir de la constatación de un muy disímil e incluso opuesto dinamismo económico de esas regiones contiguas -la "paradoja patagónica" - constatado en un periodo de cuatro décadas correspondiente a la implementación del nuevo modelo de mercado en Chile, se derivan otras paradojas adicionales que trascienden los casos referidos:

a) que tras la liberalización y el protagonismo privado persiste la injerencia -más por acción y menos por omisión- del Estado, no solamente a través de una fuerte presencia directa (geopolítica) en esos territorios, sino también mediante políticas nacionales de tanta o más incidencia que las formuladas específicamente para tales regiones;

b) que las políticas territoriales resultan menos decisivas que las políticas no territo- riales (aespaciales o supuestamente neutras en términos territoriales) especialmente las macroeconómicas, las que pueden hasta contradecirlas incluso dentro de un mismo programa gubernamental;

c) que el mayor crecimiento de las 'regiones-commodity", sobre todo las mineras, no logra compensar el rezago o menor dinamismo de las regiones-domésticas, generando una desigualdad geográfica de oportunidades y una reasignación territorial de factores productivos, incluido el trabajo, imponiendo una reconversión de las economías locales y sus mercados laborales;

d) que junto a la desindustrialización y terciarización de algunas regiones y ciudades se produce una 'reprimarización' de otras -incluso un neoextractivismo estatal- roles que, más allá de su complementariedad económica, inciden en un incremento de la desigualdad agregando, a sus factores más estructurales, si acaso no un determinismo geográfico, sí un condicionamiento;

e) que el nuevo modelo $-y$ el Estado tras él- ha detonado un reordenamiento geoeconómico y geosocial, con 'territorios de exportación' commoditizados y plenamente globales, y otros de economía más cerrada: los unos más afines y funcionales al modelo y más exitosos, mientras que los otros, más rezagados, confirman la heterogeneidad -por no decir la segregación- socioterritorial a escala regional y macrorregional;

f) que mientras los primeros, a pesar de su mayor dinamismo, están más directamente expuestos a los ciclos boom-bust de los mercados externos y a las crisis internacionales, y por ende son más vulnerables a ellas, los segundos, lejos de estar exentos de sus costos, los asumen por contagio y desplazamiento geográfico;

g) que como consecuencia de todo lo anterior el modelo- con sus políticas públicas- se ha traducido en una muy desigual e inequitativa geografía socioeconómica, menos evidente en los reconocidamente exitosos resultados e indicadores 'promedio' del país, inequidad que, a pesar de los esfuerzos redistributivos, las políticas focalizadas y los subsidios localizados, tiende a ser persistente; 
h) que, además, atendido el menor tamaño de la economía chilena y su necesaria apertura e inserción global -y su exposición a la relativa volatilidad de la inversión externa en periodos de crisis- sus territorios quedan más afectos no solo ante las decisiones estratégicas de las empresas multinacionales, sino sobre todo frente a las políticas de los organismos financieros supranacionales y a la geopolítica macroeconómica global de los mismos y de las grandes potencias;

i) en fin y en síntesis, que la muy local y aislada 'paradoja patagónica' en los confines del mundo, lejos de ser inmune a la globalización, no hace sino expresar una fuerte y muy desigual interdependencia global-local, es decir, una auténtica 'paradoja glocal' en medio de una economía mundial y una gobernanza planetaria.

\section{Referencias bibliográficas}

AALBERS, M. (editor). Subprime Cities. The political economy of mortgage markets. Malden: Wiley-Blackwell, 2012.

ALESSANDRINI, P.; FRATIANNI, M. \& ZAZZARO, A. (editors). The changing geography of banking and finance. S/L: Springer, 2010.

ÁlVAREZ, R. y FUENTES, J.R. El "síndrome holandés": teoría y revisión de la experiencia internacional. Economía Chilena, 2006, Vol. 9, № 3, p. 97-108.

BANCO CENTRAL DE CHILE. Indicadores Económicos y Sociales Regionales de Chile, 1980-2010. 2012. Disponible en Internet:

http://www.bcentral.cl/publicaciones/estadisticas/informacion-integrada/iei14.htm

BANCO CENTRAL DE CHILE. ¿Qué son las Cuentas Nacionales?. 2013. Disponible en Internet: http://www.bcentral.cl/estadisticaseconomicas/metodologias-estadisticas/cn.htm

BEINE, M. \& COUlOMBE, S. Regional perspectives on dollarization in Canada. Journal of Regional Science, 2003, Vol. 43, No 3, p. 541-569.
BRAVO, H.; GARCÍA, C.; MIES, V. y TAPIA, M. Heterogeneidad de la transmisión monetaria: efectos sectoriales y regionales. Economía Chilena, 2003, Vol. 6, № 3, p. 5-26.

BRENNER, N. Globalisation as reterritorialisation: the re-scaling of urban governance in the European Union. Urban Studies, 1999, Vol. 36, No 3, p. 431-451.

BRENNER, N. New State Space. Urban governance and the rescaling of statehood. New York: Oxford University Press, 2004.

BRENNER, N. \& ELDEN, S. (editors). State, Space, World. Selected Essays. Henri Lefebvre. Minneapolis: University of Minnesota Press, 2009.

BRENNER, N. \& THEODORE, N. Preface: From the new localism to the spaces of neoliberalism. In: BRENNER, N. \& THEODORE, N. (editors). Spaces of neoliberalism. Urban restructuring in North America and Western Europe (v-ix). Malden: Blackwell, 2002a.

BRENNER, N. \& THEODORE, N. Cities and the geographies of "actually existing neoliberalism". In: BRENNER, N. \& THEODORE, N. (editors). Spaces of neoliberalism. Urban restructuring in North America and Western Europe (v-ix). Malden: Blackwell, 2002b, p. 2-32.

CARRIÉRE-SWALLOW, Y. \& CLARO, S. Cueste lo que cueste. La batalla por salvar el Euro y Europa. Santiago de Chile: Ediciones UC, 2013.

CASTELLS, M. Redes de indignación y esperanza. Los movimientos sociales en la era de internet. Madrid: Alianza Editorial, 2013.

CORDEN, W. \& NEARY, J.P. Booming sector and the de-industrialization in a small open economy. Economic Journal, 1982, Vol. $92, N^{\circ} 368$, p. 825-848.

CORPATAUX, J.; CREVOISIER, O. \& THEURILLAT, T. The expantion of the finance industry and its impacts on the economy: a 
territorial approach based on Swiss pension funds. Economic Geography, 2009, Vol. 85, $\mathrm{N}^{\circ} 3$, p. 313-334.

DAHER, A. Políticas macroeconómicas, tipo de cambio y desarrollo territorial. Escenarios chilenos. En: ALBURQUERQUE, F.; DE MATTOS, C. \& JORDÁN, R. (editores). Revolución tecnológica y reestructuración productiva: impactos y desafíos territoriales. Buenos Aires: ILPES/ONU, IEU/PUC, GEL, 1990, p. 457-478.

DAHER, A. Regiones- commodities. Crisis y contagio en Chile. EURE, 2003, Vol. XXIX, No 86, p. 89-108.

DAHER, A. Territorios de la financiarización urbana y de las crisis inmobiliarias. Revista de Geografía Norte Grande, 2013, Nº 56, p. 7-30.

DAHER, A. Impactos territoriales de la financiarización pública postcrisis. Revista de Ciencias Sociales, Segunda Época, 2014, Vol. $6, \mathrm{~N}^{\circ} 25$, p. $13-26$.

DYMSKY, G. The global financial customer and the spaciality of exclusion after the "end of geography". Cambridge Journal of Regions, Economy and Society, 2009, N N 2, p. 267-285.

ESCOBAR, B. \& REPETTO, A. Efectos de la estrategia de desarrollo chilena en las regiones: una estimación de la rentabilidad del sector transable regional. Colección Estudios CIEPLAN, 1993, No 37, p. 5-36.

GONZÁLEZ, R. Poderes locales, nación y globalización. Santiago de Chile: Universidad Academia de Humanismo Cristiano, 2007.

GONZÁLEZ, S. La geografía escalar del capitalismo actual. Scripta Nova. Revista de Geografía y Ciencias Sociales, 2005, Vol. IX, $\mathrm{N}^{\circ} 189$, p. $1-21$.

GUERRERO, S. \& POZO, M.P. Empalme estadístico del PIB y de los componentes del gasto: Series anuales y trimestrales 19862003, Referencia 2008. Santiago de Chile: Estudios económicos estadísticos Banco Central de Chile, 2012, No 91. Disponible en Internet: http://www.bcentral.cl/estudios/estudioseconomicos-estadisticos/pdf/see91.pdf

FEDERAL RESERVE SYSTEM. Beige Book. Washington DC: Board of Governors of the Federal Reserve System, 2012.

HARVEY, D. The limits of capital. Chicago: University of Chicago Press, 1982.

HARVEY, D. Espacios del capital. Hacia una geografía crítica. Madrid: Ediciones Akal, 2007.

HARVEY, D. Las raíces urbanas de las crisis financieras. En: BELIL, M.: BORJA, J. y CORTI, M. (editores). Ciudades: una ecuación imposible. Buenos Aires: Ed. Café de las Ciudades, 2012a, p. 321-358.

HARVEY, D. The urban roots of the financial crisis. In: AALBERS, M. (editor). Subprime Cities. The political economy of mortgage markets. Malden: Wiley-Blackwell, 2012b, p. xiii-xix.

HARVEY, D. Ciudades rebeldes. Del derecho a la ciudad a la revolución urbana. Madrid: Ediciones Akal, 2013.

JONAS, M. New Institutional Spaces. London: Jessica Kingsley, 1999.

KENNEN, P. The theory of optimum currency areas: an eclectic view. In: MUNDELL, R. \& SWOBODA, A. (editors). Monetary problems of the international economy. Chicago: The University of Chicago Press, 1969, p. 41-68.

LEYSHON, A. Money/Space: Geography of monetary transformation. London: Taylor and Francis, 1997.

MANYKA, J.; REMES, J.; DOBBS, R; ORELLANA, J. \& SCHAER, F. Urban America: US cities in the global economy. New York: McKinsey Global Institute, 2012.

MARTIN, R. Money and the space economy. New Jersey : Wiley, 1998.

MARTIN, R. The local geographies of the financial crisis: from the housing bubble 
to economic recession and beyond. Journal of Economic Geography, 2011, No 11, p. 587-618.

MCKINNON, R. Optimum currency areas. American Economic Review, 1963, Vol. 53, $\mathrm{N}^{\circ} 4$, p. $717-725$.

MUNDELL, R. A theory of optimum currency areas. American Economic Review, 1961, Vol. 51, № 3, p. 657-665.

OBSERVATORIO REGIONAL SUBDERE. 2013. Disponible en Internet: http:// www.subdere.gov.cl/organizaci\%C3\%B3n/ divisi\%C3\%B3n-de-pol\%C3\%ADticas$y$-estudios/departamento-de-estudios-yevaluaci\%C3\%B3n/observatorio-reg

O'BRIEN, R. Global financial integration: the end of geography. London: Chatham House Papers, 1992.

PECK, J. \& TICKELL, A. Neoliberalizing space. In: BRENNER, N. \& THEODORE, N. (editors). Spaces of neoliberalism. Urban restructuring in North America and Western Europe (v-ix). Malden: Blackwell, 2002, p. 33-57.

PEREIRA, M.; ULLOA, A.; O'RYAN, R. y DE MIGUEL, C. Síndrome holandés, regalías mineras y políticas de gobierno para un país dependiente de recursos naturales: el cobre en Chile. Santiago de Chile: CEPAL, Medio Ambiente y Desarrollo, 2009, № 140.

PERLO, M. Cities in times of crisis: The response of Local Governments in light of the global economic crisis. Berkeley: IURD Working Paper Series 2011-01UC Berkeley, 2011.

RAJAN, R. \& RANCHARAM, R. Tierras en venta. Finanzas y Desarrollo, 2011, Vol. 48, $\mathrm{N}^{\circ} 4$, p. 30-33.

RODRÍGUEZ-POSE, A. y PETRAKOS, G. Integración económica y desequilibrios territoriales en la Unión Europea. EURE, 2004, Vol. XXIX, No 89 , p. 63-80.
SASSEN, S. Contrageografías de la globalización. Género y ciudadanía en los circuitos transfronterizos. Madrid: Traficantes de sueños, 2003.

SASSEN, S. El reposicionamiento de las ciudades y regiones urbanas en una economía global: ampliando las opciones de políticas y gobernanza. EURE, 2007, Vol. XXXIII, № 100, p. 9-34.

SASSEN, S. Territorio, autoridad y derechos. De los ensamblajes medievales a los ensamblajes globales. Buenos Aires: Katz, 2010.

SASSEN, S. Expanding the terrain for global capital: When local housing becomes an electronic instrument. In: AALBERS, M. (editor). Subprime Cities. The political economy of mortgage markets. Malden: Wiley-Blackwell, 2012, p. 74-96.

SHEPPARD, E. Geographical political economy. Journal of Economic Geography, 2011, № 11, p. 319-331.

SOKOL, M. Towards a "newer" economic geography? Injecting finance and financialisation into economic geographies. Cambridge Journal of Regions, Economy and Society, 2013, No 6, p. 501-515.

THEURILLAT, T. The negotiated city: between financialisation and sustainability. Neuchätel: Research Group in Territorial Economy, University of Neuchätel, 2009.

VAN OVERTVELDT, J. O Fim do Euro: A história da moeda da Uniäo Europeia e seu futuro incerto. Rio de Janeiro: Elsevier, 2012. 